\title{
Angoscia esistenziale, attese di salvezza e nostalgia di Dio nella cinematografia di Ingmar Bergman
}

\author{
Giovanni Amendola \\ Dipartimento di Matematica e Informatica \\ Università della Calabria \\ amendola@mat.unical.it
}

Abstract: In this article, we will analyze from an existentialist-soteriological perspective three of Ingmar Bergman's cinematographic masterpieces, which are placed between the late fifties and the early sixties of the last century: Wild Strawberries (1957), Through a Glass Darkly (1961) and Winter Light (1963). Our reflection will be accompanied by some fundamental questions. Is it possible to find expectations, or at least a nostalgia for salvation, starting from the existential anxiety of the protagonists of these films? Does their sense of nausea towards a life that seems to sink into the most distressing oblivion still leave a glimmer towards some light that can penetrate to restore hope? Is it possible to recognize a need for salvation from death and beyond death itself? We will try to show how the request for salvation declines first and foremost as salvation in this life through the search for interpersonal relationships, in the perspective of living for others and in the possibility of loving for free, in a constant struggle in the face of existential anxiety that seems to continue to loom and could still have the upper hand. Finally, when salvation is understood as stability in a life at the mercy of the waves of reality, it is recognized in the presence of God as warrant of the authenticity of every act and desire for love.

Keywords: Existentialism, Soteriology, Ingmar Bergman, Salvation, Nostalgia, Theology

Sommario: In questo articolo, analizzeremo da una prospettiva esistenzialistasoteriologica tre dei capolavori cinematografici di Ingmar Bergman, che si collocano tra la fine degli anni Cinquanta e l'inizio degli anni Sessanta del secolo scorso: Il posto delle fragole (1957), Come in uno specchio (1961) e Luci d'inverno (1963). La nostra riflessione sarà accompagnata da alcune domande fondamentali. È possibile rinvenire, a partire dall'angoscia esistenziale dei protagonisti di questi film, delle attese o, perlomeno, una nostalgia di salvezza? Il loro senso di nausea verso una vita che sembra sprofondare nell'oblio 
più angosciante lascia ancora aperto uno spiraglio verso una qualche luce che può penetrare per ridare speranza? È possibile ravvisare un bisogno di salvezza dalla morte e oltre la morte stessa? Cercheremo di mostrare come la richiesta di salvezza si declini innanzitutto come salvezza in questa vita attraverso la ricerca di relazioni interpersonali, nella prospettiva del vivere per gli altri e nella possibilità di amare gratuitamente, in una costante lotta di fronte all'angoscia esistenziale che sembra continuare a incombere e potrebbe ancora avere la meglio. Infine, quando la salvezza viene intesa come stabilità in una vita in balia delle onde del reale, essa viene riconosciuta nella presenza di Dio come garante dell'autenticità di ogni atto e desiderio di amore.

Parole chiave: Esistenzialismo, Soteriologia, Ingmar Bergman, Salvezza, Nostalgia, Teologia

\section{INTRODUZIONE}

Il disincanto di un progresso umano affidato all'avanzamento del mondo tecno-scientifico e l'illusione delle ideologie neopositiviste sono lo sfondo in cui l'uomo ritrova la sua esistenza sganciata da certezze prima ritenute inattaccabili ed emergono interrogativi che si slanciano su un abisso mai colmato. È un ritorno alla realtà autentica troppo spesso abbandonata all'astrattezza di una razionalità fredda, non più metafisica, ma estremamente logico-deduttiva. È l'emergere di un pensiero altro, che era stato fagocitato dall'incalzare di una modernità fondata sul razionalismo cartesiano. Una sorta di esplosione dall'interno del pensiero occidentale attraverso, particolarmente, quella corrente di pensiero che prenderà il nome di esistenzialismo.

Uno dei suoi massimi esponenti come Martin Heidegger, parallelamente all'esaltazione neopositivista del pensiero logico-formale delle scienze allora ritenute esatte ${ }^{1}$, svilupperà una critica profonda di tale deriva della ragione umana verso ciò che, nei suoi termini, nulla ha a che fare con l'autenticità del pensiero, fino a poter sostenere la tesi paradossale e categorica secondo

1 Cf. H. Hann y otros, La concezione scientifica del mondo. Il circolo di Vienna (Laterza, Roma-Bari 1979). 
cui "la scienza non pensa" ${ }^{2}$. Secondo il filosofo tedesco, pensare richiede un salto qualitativo che si pone al di là del metodo delle scienze empiriche. Il salto nel pensiero porta con sé un carattere intrinsecamente nuovo e sorprendente a tal punto che, con un gioco di parole, si arriva a identificare il pensare (Denken) con il ringraziare (Danken). Si tratta di un pensiero che varca il limite, che compie un salto, in un territorio non più chiaro e distinto, ma incerto e indefinito, a cui tuttavia non è lecito sottrarsi a meno di non rinunciare a priori a ciò che darebbe appunto da pensare.

L'emergere di questa dimensione in gran parte assopita, almeno sul panorama filosofico, tocca le profondità dell'essere umano che non può che ritrovarsi come spaesato, specie in contrapposizione alle certezze propugnate delle scienze positive, che tuttavia non riescono a offrire risposte a tali questioni. O perché le escludono a priori dal proprio campo di indagine ritenendole prive di significato oppure perché le approcciano con metodologie che, forse, possono offrire delle risposte a livello esistentivo (existentiell), ma non a quello esistenziale (existential) ${ }^{3}$. È a questo livello che l'uomo percepisce se stesso come essere storico e progettuale, in cui tuttavia la realizzazione ipoteticamente crescente del suo esserci si scontra con una temporalità destinata a decrescere, fino ad annullare ogni ulteriore progettualità. Tale pensiero è reso possibile per mezzo del sentimento dell'angoscia. Sebbene, in tale contesto, l'angoscia si possa considerare come una finestra del pensiero filosofico sulla costante presenza della morte e, quindi, dell'annientamento dell'esserci, da una prospettiva più psicologica, ciò può tradursi in angoscia esistenziale, paralizzando l'agire umano e degenerando in disperazione $e^{4}$. L'angoscia esistenziale, nella sua forma più radicale, si connette a una introspezione per certi versi solipsistica, dove il soggetto è solo con se stesso in totale solitudine. La realtà, in tal modo, viene costantemente deformata e le relazioni intersoggettive risultano irrisorie, incapaci di sostenere il confronto con l'io del soggetto.

L'angoscia, la solitudine esistenziale e il dramma di una soggettività proiettata verso il suo annientamento sono indagati in modo eminente dal regista svedese Ingmar Bergman. I personaggi dei suoi film sono caratterizzati da una profonda analisi del vissuto psicologico, emergente attraverso un'attenta

2 M. Heidegger, Che cosa significa pensare? (SugarCo, Milano 1971) 41.

3 Cf. M. Heidegger, Essere e tempo (Longanesi, Milano 1976) 32-35.

4 Cf. S. KierkegaArd, Il concetto dell'angoscia. La malattia mortale (Sansoni, Firenze 1953). 
cura dei particolari visivi e sonori. Un'esteriorizzazione che, in diverse circostanze, rispecchia il vissuto interiore ed esistenziale dello stesso regista ${ }^{5}$. La tematica esistenziale-religiosa permea infatti i suoi film.

Il regista era figlio di un pastore protestante e la sua educazione religiosa, come egli stesso racconta, era basata "sui concetti di peccato, confessione, punizione, perdono e grazia, fattori concreti nelle relazioni dei bambini con i genitori e con Dio"6. Il rigido sistema educativo commisto ad alcune situazioni drammatiche familiari, porteranno il giovane Ingmar a duri scontri con il padre e alla ribellione verso quel sistema valoriale, dal quale si allontanerà fino ad affermare: "Io non sono un credente. Qualsiasi forma di salvezza ultraterrena mi suona blasfema. Per farla breve: la mia vita è priva di significato" ". La categoricità di tali affermazioni potrebbe far trasparire una chiusura pregiudizievole verso la fede religiosa. Ma crediamo che il cammino di Bergman verso tale approdo sia ben più ponderato e, per certi versi, sempre incompiuto. D'altronde se le domande esistenziali restano aperte, come accade nei suoi film, rimane costante la stessa ricerca di senso da attribuire ad una vita che avanza inesorabilmente verso la morte.

Sebbene Bergman giunga ad affermare che una salvezza ultraterrena gli sembra impossibile, riteniamo invece che sia ancora possibile indagare nei suoi film ciò che, in qualche modo, richiama o semplicemente lascia emergere un bisogno di salvezza. Ci soffermeremo esclusivamente sull'analizzare da tale prospettiva soteriologica tre dei suoi capolavori cinematografici che si collocano tra la fine degli anni Cinquanta e l'inizio degli anni Sessanta del secolo scorso: Il posto delle fragole (1957), Come in uno specchio (1961) e Luci d'inverno (1963). La nostra riflessione sarà accompagnata, pertanto, da alcune domande fondamentali. È possibile rinvenire, a partire dall'angoscia esistenziale dei protagonisti di questi film, delle attese o, perlomeno, una nostalgia di salvezza? Il loro senso di nausea verso una vita che sembra sprofondare nell'oblio più angosciante lascia ancora aperto uno spiraglio verso una qualche luce che può penetrare per ridare speranza? È possibile ravvisare un bisogno di salvezza dalla morte e oltre la morte stessa?

5 Cf. I. Bergman, Lanterna magica (Garzanti, Milano 1987).

6 I. Bergman, Lanterna magica, 12.

7 Tale affermazione si ritrova in un dattiloscritto del 1974 presente negli archivi della Rai in riferimento alla proposta fattagli per girare un film su Gesù, quello che poi sarà invece affidato a Franco Zeffirelli (cf. V. FanTuZzI, "In memoria di Ingmar Bergman", in La Civiltà Cattolica 3776/4 [2007] 153-166). 


\section{UNA SALVEZZA DALLA SOLITUDINE ESISTENZIALE}

Il primo film che poniamo all'attenzione è Il posto delle fragole del 1957. Il protagonista è un anziano medico, Isak Borg, che vive a Stoccolma. Ha concluso la sua brillante carriera professionale e sta per essere insignito di un prestigioso riconoscimento accademico a Lund.

Fin dall'inizio emerge il suo disagio esistenziale che si acuisce nell'avvertire sempre più insistentemente l'avvicinarsi della propria morte. Il primo dei quattro sogni che rivelano le inquietudini del protagonista, lo vede vagare in una città in cui gli orologi sono senza lancette, quasi a indicare che il tempo giunge al suo termine. Ancor più eloquente è la comparsa di un carro funebre che urtando su un lampione provoca la caduta a terra di una bara e la sua apertura. Un braccio fuoriesce e lo afferra, lo tira con forza a sé e, nell'avvicinarlo, Isak riconosce nel morto il suo stesso volto. Svegliatosi decide di non partire con l'aereo come programmato, ma di intraprendere il viaggio in auto. La governante non è d'accordo con l'iniziativa del professor Isak ma, suo malgrado, gli prepara la valigia. Al viaggio in macchina si unisce la nuora Marianne, che sta vivendo con il suocero e ora vuole recarsi dal marito Evald. Coglie così l'opportunità, altrimenti per motivi economici non sarebbe potuta partire.

Durante il viaggio Marianne pone brutalmente Isak di fronte alla sua realtà: non è il professore tanto acclamato per le sue grandi opere di bene verso l'umanità, ma una persona fredda ed egoista, senza sentimenti, ormai destinata alla solitudine e alla morte. Temi che continueranno a ricorrere incessantemente nel prosieguo del viaggio.

Nel tragitto Isak decide di fare una deviazione e conduce la nuora in un luogo a cui è molto legato per i ricordi della sua gioventù: il posto delle fragole. È qui che si riaffacciano, come in un secondo sogno, i ricordi del suo primo amore, sua cugina Sara, che poi lo lascerà per suo fratello Sigfrid. Nel ritorno alla realtà, Isak è risvegliato da una giovane ragazza, interpretata dalla stessa attrice di Sara e avente il suo stesso nome. Gli chiede un passaggio, poiché è diretta verso l'Italia con altri due suoi amici. Il viaggio riprende.

I cinque si ritrovano così a mangiare assieme e a parlare dell'esistenza di Dio. Si scaldano gli animi tra i due amici e spasimanti della ragazza. Uno è studente di teologia, l'altro invece di medicina. Il primo è credente, il secondo è ateo. Si accusano rispettivamente di razionalismo e di fantasia. $\mathrm{La}$ 
domanda viene così rimbalzata al professore Isak che però evita di rispondere direttamente per non entrare in una disputa che ritiene non porti a nulla. Preferisce invece ricordare una poesia, aiutato dagli altri commensali:

Dov'è l'amico che il mio cuore ansioso ricerca ovunque senza aver mai riposo? Finito il dì ancor non l'ho trovato e resto sconsolato. La sua presenza è indubbia e io la sento in ogni fiore e in ogni spiga al vento.

L'aria che io respiro e dà vigore del suo amore è piena.

Nel vento dell'estate la sua voce intendo ${ }^{8}$.

In seguito i due giovani arriveranno persino a lottare per l'esistenza di Dio. La domanda continua a riecheggiare: "Esiste o no?", stavolta nella sottile ironia di Sara, che lascia intendere di ritenere inutile e una perdita di tempo un tale interrogativo. Ma come in precedenza, nessuna risposta giunge e l'interrogativo cade nel silenzio, poi coperto dal successivo irrompere di un fragoroso temporale.

Durante una pausa nel viaggio, Isak sogna per la terza volta. È di nuovo nel posto delle fragole con la sua prima amata, Sara, che mostrandogli uno specchio, con freddezza, gli dice: "Guarda cosa sei! Un vecchio timoroso che presto morirà!". Emerge sempre più la delusione e il dolore causati da Sara che decide di lasciarlo per sposare il fratello. Gli viene così rinfacciato che tutta la sua arte medica non può far nulla per comprendere quel dolore che lo trafigge. La spiegazione chimico-biologica può forse descriverlo, ma è insufficiente a comprenderlo. Il contrasto è fortissimo di fronte all'imminente riconoscimento accademico.

8 Tale poesia, che più propriamente è una sorta di salmo, è ripresa da un testo dell'arcivescovo luterano svedese vissuto nel xIX secolo, Johan Olof Wallin (Stora Tuna 1779 - Uppsala, 1839). Il titolo originale è Var är den Vän som överallt jag söker (Dove è l'Amico che cerco ovunque). Il salmo fu composto il 4 luglio 1818 e si ritrova al termine di un discorso pronunciato da Wallin in occasione dell'inaugurazione di un nuovo luogo di sepoltura per le parrocchie di Västerås e St. Ilians. Fu pubblicato nel libro dei Salmi di Wallin nel 1819 come numero 481. In questa prima edizione la traduzione letterale dei primi quattro versetti è la seguente: "Dove è l'Amico, che ovunque sto cercando? / Quando il giorno cresce, la mia voglia aumenta soltanto; / Quando il giorno vola, non lo trovo mai, / bruciature fisse del cuore" (cf. https://sv.wikipedia.org/wiki/Var_\%C3\%A4r_den_V\%C3\%A4n,_som_\%C3\%B6verallt_jag_s\%C3\%B6ker). 
Il sogno continua e Isak si ritrova a dover sostenere un esame/giudizio. A giudicarlo sono le persone che ha incontrato nella sua vita. Gli vengono poste delle domande, ma a nessuna riesce a rispondere. Gli viene chiesto di leggere una scritta sulla lavagna, ma sono per lui parole prive di significato. Nonostante la traduzione dell'esaminatore: "Qual è il primo dovere di un medico?”, Isak riconosce di non sapere la risposta. Questa invece risuona nel timbro duro dell'esaminatore, manifestando in Isak un interiore bisogno di riconciliazione: "Il primo dovere di un medico è di chiedere perdono!". Il verdetto finale è inequivocabile: "Lei è un incompetente". Inoltre viene accusato di altri errori: "indifferenza, egoismo, incomprensione". Sono accuse rivolte dalla defunta moglie Karin, celate nell'atteggiamento razionalistico e insensibile del marito nell'affrontare il tradimento di lei: "non gliene importa niente perché è un essere gelido". Infine il severo insegnante decreta la punizione finale, che è, a suo dire, quella usuale, cioè la solitudine. Allora una domanda riecheggia nella mente di Isak nel risvegliarsi dal sonno: "Non vi sarà clemenza?”.

Inizia così un nuovo discorso con la nuora dove il dottore mette a nudo il suo io: "Sono morto pur essendo vivo". Non sono parole nuove per Marianne perché anche il marito Evald non trova nessuna motivazione valida per continuare a vivere e perché una nuova vita possa nascere. Di fatto rifiuta il bimbo che sua moglie porta in grembo: "Intorno solo freddo, morte e solitudine ovunque". Evald non trova attorno a sé nessuna speranza, per questo ha freddamente scelto di non consegnare a tale mondo un altro uomo. Marianne è stata così posta di fronte al bivio di separarsi dal marito o di rinunciare al nascituro. Ha tuttavia deciso che il bambino dovrà nascere, nonostante l'amore che sente per Evald, sta andando da lui per annunciargli la sua decisione. Il senso di nausea verso la vita da parte di Evald e la sensazione di vivere come se fosse morto di Isak è paradossalmente enfatizzata nel sopraggiungere gioioso dei tre giovani che, avendo saputo del suo prossimo riconoscimento accademico, hanno raccolto per lui un mazzo di fiori e lo acclamano come un uomo saggio che ha vissuto una vita felice acuendo il contrasto nell'interiorità del protagonista.

Il viaggio giunge finalmente al termine. La sua governante lo attende a Lund dove è presente anche il figlio Evald. La cerimonia accademica è la celebrazione dell'apparenza e dell'inautenticità. Il rituale viene estremizzato fino al ridicolo attraverso squilli di tromba, imposizione di oggetti simbolici, cappello e anello, ed un solenne latino. 
Giunta la sera Isak, in diversi incontri, manifesta concretamente qualcosa di nuovo. Alla governante si rivolge in tono colloquiale: "Non potremmo darci del tu?". Ma le convenzioni sociali producono nella governante un categorico rifiuto. Sotto la finestra della sua camera i tre giovani gli dedicano una serenata e un affezionato saluto, in particolare, da parte della ragazza controfigura della sua prima amata: "Addio papà Isak! Sei il grande amore della mia vita! Oggi, domani e per tutta l'eternità!". A cui Isak risponde con voce bassa quando ormai sono troppo lontani: "Scrivetemi qualche volta". Poi è il momento di incontrare Evald al quale mostra l'interesse verso un esito positivo della relazione con Marianne, sebbene non riesca a esprimere quanto vorrebbe comunicare al figlio, ovvero l'andare oltre ad una intransigente regola di comportamento. Infine si sofferma proprio con la nuora, dicendole di volerle un gran bene e ricevendo da lei un caloroso grazie. Il film si conclude col quarto ed ultimo sogno. Isak si addormenta ripensando ai periodi felici dell'infanzia, dove trova conforto e serenità nel ritrovare e rivedere, come in una bucolica icona, i suoi genitori.

È presente in Isak una qualche nostalgia di salvezza? La risposta ci sembra affermativa. Isak avverte la sua vita come un fallimento. Sembra rimanere soltanto l'ultima possibilità dell'esistere umano: la morte. È questa che lo chiama continuamente e lo invita ad un ripensamento. Si tratta di passare dall'inautentico, le acclamazioni e gli appariscenti elogi, all'autenticità di una vita che rivede se stessa alla luce della propria fine. È da lì che parte il viaggio. Un viaggio esistenziale che pone Isak alla ricerca di una soluzione a quel bisogno impellente di autenticità. Potremmo dunque in prima analisi parlare di nostalgia di autenticità e, quindi, di nostalgia di vita autentica. E questa vita autentica non è forse quella saggezza e felicità che proprio quei ragazzi, nella loro semplicità, reclamano nel loro amato professore? Una felicità che si declina nel ribaltare la sentenza finale della sua condanna: dalla solitudine alla ricerca di relazioni. Sono queste infatti che lo giudicano e lo condannano. È dunque per Isak il momento del "disgelo". La scoperta di un bisogno di relazioni autentiche mette in cammino concreto l'anziano dottore. Cerca di sentire ed entrare in simpatia con gli altri volti della sua vita: la governante, i tre ragazzi, il figlio Evald e la nuora Marianne. $\mathrm{Ha}$ compreso profondamente che saranno in un certo qual modo essi a giudicarlo, o meglio, la qualità delle relazioni potranno superare l'angoscia della solitudine esistenziale. 
La domanda a questo punto che ci sembra emergere è la seguente: questa solitudine che può essere allontanata o quanto mai attenuata da nuove relazioni può davvero annientare quella morte che inarrestabilmente prosegue la sua corsa? C'è una risposta a questa domanda da parte di Isak? Una risposta direttamente affermativa o negativa non ci sembra riscontrabile nel film. Forse anche per non sciogliere la forza vitale di un tale interrogativo, che non può non ricondurci alla possibilità di una salvezza anche dalla morte e attraverso la morte stessa. $\grave{E}$ in ultima analisi la domanda sull'esistenza di Dio, come colui che salva nella morte. Come osservato nella trama, si tratta di un argomento che percorre tutto il viaggio e si fa presente ogni qual volta compaiono i due giovani contendenti. Per Isak sembra non esserci una risposta che proceda da un argomentare esclusivamente razionale. Diremo quasi che, in analogia col pensiero heideggeriano, Isak incontra lì un "sentiero interrotto" (Holzwege) 9 e solo l'intensità poetica può balbettare qualcosa di una presenza che è sempre oltre, ma che tutto avvolge e continuamente chiama alla sua amicizia.

\section{SOLO L'AMORE CI PUÒ SALVARE}

Il secondo film che prendiamo in considerazione è intitolato Come in uno specchio. Riprende così la prima lettera ai Corinzi di san Paolo: "Adesso noi vediamo come in uno specchio, in maniera confusa; allora vedremo faccia a faccia" $(1 \operatorname{Cor} 13,12)$. Lo specchio a cui si riferisce Paolo non è per nulla simile ai nostri specchi, realizzati, grazie a determinati materiali e tecnologie di taglio, con superfici estremamente piane che riflettono con chiarezza la realtà fisica che hanno di fronte. Si tratta piuttosto di superfici metalliche, in cui era inevitabile che la struttura fosse a curvatura non nulla e, pertanto, deformasse i corpi che in esso si rispecchiavano, confondendone il vero aspetto e le reali forme. Il film è dominato da questa ambivalenza tra mondo reale e mondo ideale, messa in luce attraverso la tematica della malattia e della sofferenza umana.

Sono quattro i protagonisti dell'opera: Martin e Karin, una coppia di sposi; Minus, fratello di Karin e David loro padre. Si trovano a trascorrere un periodo di vacanza su un'isola, dopo che Karin è stata rimessa dall'ospedale psichiatrico dove era ricoverata per le sue crisi psicotiche. Inizialmente Karin si mostra alquanto serena, ma molto infantile nei modi di fare, mani- 
festando un certo disagio nello stare col marito Martin, al quale preferisce invece la compagnia del fratello di 17 anni, che comincia ad affacciarsi su una realtà del mondo che ancora non aveva sperimentato, si trova di fatto in una fase a cavallo tra il mondo della spensieratezza fanciullesca e quello incalzante che "spezza il cerchio", per usare una espressione che pronuncerà più avanti David, attraverso esperienze di sofferenza e di morte, di ricerca di senso e di significati più profondi, che dovrebbero rispondere a tale inarrestabile incedere di una realtà altra da quella addomestica dal pensiero.

Durante la notte tuttavia si manifestano i sintomi della malattia di Karin. Si reca in solaio perché lì sente delle voci che la chiamano. Va poi nella camera del padre. David cerca di tranquillizzare la figlia e la fa dormire nel suo letto, mentre lui è intento a scrivere il suo ultimo romanzo. Sopraggiunge intanto il figlio Minus che lo invita ad andare in barca a tirare le reti. David allora lascia tutti i suoi fogli sulla scrivania ed esce con Minus.

Karin si risveglia e, frugando tra le carte del padre, in un cassetto, trova un diario dove legge che la sua malattia è senza alcuna speranza di guarigione. Ma ciò che ne distrugge ancor più il suo animo fragile è la strumentalizzazione che ne fa il padre: "Con spavento constato la mia curiosità, l'impulso di prendere nota dei sintomi, di registrare giorno per giorno il graduale disfacimento di mia figlia, di usufruirne e sfruttarla”. Karin va così a svegliare il marito. Gli racconta solo la prima parte di quanto ha letto perché della seconda non riesce neppure a parlarne.

Al mattino David e Martin escono in barca, mentre Karin e Minus rimangono sull'isola. Minus confida alla sorella i suoi dubbi esistenziali: "Mi domando se tutti vivano chiusi in se stessi [...] nel proprio mondo. Tu nel tuo. Io nel mio. Ognuno la propria cella". Karin invece sconvolge Minus con le sue confessioni. Infatti crede che Dio stesso dovrà apparire a lei a agli altri che vede oltre la parete nel solaio: "La malattia era come un sogno. Ma questo non può essere un sogno. Deve essere la realtà! Deve essere la realtà! [...] A volte mi trovo in questo mondo a volte nell'altro, senza che io possa impedirlo". Minus l'ascolta incredulo e comprende la gravità dello stato in cui si trova la sorella. Vorrebbe aiutarla, ma non sa come.

Sulla barca intanto si consuma un altro intenso dialogo tra David e Martin. Quest'ultimo vuole sapere interamente ciò che David ha scritto su Karin nel suo diario e, dopo averlo saputo, per confessione stessa del suoce- 
ro, lo accusa per la sua spietatezza:

In ogni cosa vedi solo il tuo io. La tua insensibilità ti ha reso un essere perverso. Studiarne i sintomi? È un'espressione che ti definisce [...] C’è solo una cosa di cui non possiedi la più pallida idea: è la vita stessa [...] Diventi geniale solo quando si tratta di trovare giustificazioni e scuse [...] Una cosa mi è oscura [...] I tuoi romanzi rivelano un velato timore di Dio. Ti posso assicurare che la tua fede e i tuoi dubbi appaiono poco convincenti. Quella che invece traspare evidente è la tua genialità mostruosa [...] Perché non affronti il tuo lavoro con più umanità? [...] Ciò che non comprendo è come tu possa contaminare Dio con queste brutture. Un entità così alta e imperscrutabile.

Tuttavia David non sembra corrispondere a quanto Martin afferma. Forse così è stato in precedenza, ma qualcosa in lui sembra essere cambiato, almeno ciò è noto allo spettatore, che ha assistito all'inizio del film, al suo dolore e al suo pianto per la decisione presa in precedenza di intraprendere un nuovo lavoro in Jugoslavia, abbandonando nuovamente la sua famiglia. Ma qualcosa in lui è cambiato e ora può confessarlo al genero:

Laggiù in Svizzera avevo deciso di suicidarmi [...] Mi diressi sul precipizio a tutto gas, ma il carburante si ingolfo. Di colpo frenai come un automa [...] Mi trascinai fuori dall'auto e presi a tremare in tutto il corpo $[\ldots]$ con un senso di oppressione che mi mozzava il fiato [...] Non ho più alcuna ragione di fingere. La verità non si rivela che con le catastrofi $[\ldots]$ Dal mio animo vuoto sbocciò qualcosa che non ho quasi il coraggio di nominare. Un amore per Karin, per Minus e per te. Un giorno forse ti dirò tutto. Adesso non ne ho il coraggio. Ma se tutto sta come io spero, avremo il tempo di riparlarne.

Mentre si trova col fratello Minus, Karin ha un'altra crisi e, improvvisamente, scappa via. Minus, dopo varie ricerche in casa, la ritrova accasciata al suolo in un relitto abbandonato sulla riva del mare. Karin ha freddo e chiede una coperta, allora Minus corre a casa per prenderla, ma crolla per il dolore di fronte al letto, implorando Dio. Ė un'invocazione che racchiude la richiesta di senso di fronte ad una realtà divenuta incomprensibile: la sofferenza psichica della sorella e la sua impotenza nel poterla aiutare.

Nel frattempo giungono a riva David e Martin. Avvertiti da Minus si recano immediatamente da Karin. Martin va da lei, ma viene rifiutato dalla 
moglie, che chiede soltanto di parlare col padre al quale confessa:

Non si può vivere in due mondi. Bisogna scegliere. Non ho più la forza di passare da un mondo all'altro [...] Così non posso andare più avanti: odiando [...] Devo fare quello che la voce mi impone. Non riesco a venirne a capo [...] Papà è orrendo vedere il proprio sconvolgimento e non capirlo.

È drammatica la consapevolezza di Karin del suo stato schizofrenico. Pur riconoscendo la contraddittorietà dello sdoppiamento della realtà in cui si ritrova, non ha più alcun criterio per discernere quella che dovrebbe essere la realtà autentica. In lei, l'ambivalenza presente anche negli altri personaggi, raggiunge il vertice dell'aporia. La realtà è totalmente deforme, proprio come nello specchio a cui fa riferimento Paolo nel testo della lettera ai Corinzi. E tale deformità è accresciuta dalla distanza razionalistica degli scritti del genitore. Ora, invece, con affetto David si avvicina alla figlia e le spiega i suoi comportamenti e il suo egoismo:

Ho la nausea quando penso al tempo sacrificato alla mia cosiddetta arte. Tua mamma morì, ma il mio primo successo fu più importante della sua morte. Ne gioivo segretamente. Eppure amavo tua madre anche se in modo egoistico [...] In te vedevo la malattia di tua madre. Non feci che scappare per terminare il mio romanzo [...] Vedi Karin si traccia un magico cerchio attorno a noi escludendo ciò che può compromettere i nostri intenti. Ma, quando la vita spezza il cerchio, questi intenti si rivelano meschini e insignificanti. Così tracciamo un nuovo cerchio e un nuovo riparo.

La figlia lo ascolta e lo compatisce: "Povero papà!". E David: "Povero papà... Costretto dalla vita a vivere nella realtà". Ecco di nuovo ricomparire il tema centrale dell'opera, che dalla nostra prospettiva, potremmo declinare come una iniziale ricerca di salvezza nella fuga dal reale attraverso la costruzione teorica, astratta e ideale di un mondo duale e addomesticato dal soggetto. Tuttavia in questo mondo idealizzato a propria misura, non ci può essere salvezza, innanzitutto perché sarebbe esclusivamente il mondo del soggetto solipsisticamente inteso, dove non c'è spazio per gli altri, se non come pedine che percorrono gli schemi creati dal soggetto stesso e, poi, come riconosce lo stesso David, l'idealizzazione finirà con lo scontrarsi con la realtà autentica a cui vanamente si è tentato di sfuggire, specie nelle forme più tragiche della sofferenza e della morte. È questo il luogo dove il cerchio 
si spezza e la realtà riemerge contrastando l'idea consolidata nella mente ${ }^{10}$.

Martin nel frattempo va a chiamare l'ambulanza. Karin, tornata a casa, ha di nuovo una forte crisi. I suoi familiari la sentono parlare nel solaio. Mentre è inginocchiata sul pavimento, dicendo di attendere l'arrivo di Dio, comincia ad urlare e ad agitarsi. Riescono finalmente a bloccarla e a farle un'iniezione per calmarla. Lei è ancora sotto shock: "Il Dio che è entrato era solo un ragno $[\ldots]$ un viso ripugnante e gelido [...] voleva possedermi $[\ldots]$ ma io mi sono difesa [...] Ho visto Dio". Dopo poco giunge l'ambulanza e in elicottero Karin ritornerà all'ospedale psichiatrico.

Minus, che ha assistito a tutto il dramma della sorella, cade nuovamente in pianto. Si reca dal padre confidandogli di avere paura. Ha così inizio l'ultimo intenso dialogo del film. Le preoccupazioni e i dubbi che Minus nutre nei confronti della propria esistenza vengono esplicitati al padre, che espone al figlio le sue speranze e il senso profondo che ha rinvenuto dalle esperienze della sua vita:

Minus: "Non posso vivere in questo nuovo mondo".

David: "Sì che puoi, se avrai qualcuno su cui sostenerti".

Minus: "Chi secondo te, un Dio? Dammi una prova di Dio, non puoi". David: "Si che posso, ma devi ascoltare bene ciò che ti dico Minus".

Minus: "Si, ho bisogno d'ascoltare papà".

David: "Posso darti solo una pallida idea delle mie speranze. Dio è la certezza che l'amore esiste come cosa concreta in questo mondo di uomini". Minus: "Intendi un amore particolare, è vero?".

David: "Ogni genere d'amore: il più elevato ed il più infimo, il più oscuro ed il più splendido. Ogni specie d'amore".

Minus: "Anche il desiderio d'amore?".

David: "Il desiderio e la repulsione, miscredenza e fede".

Minus: "L'amore è una dimostrazione di Dio?".

David: “Non so se l'amore dimostri l'esistenza di Dio oppure se l'amore

10 Ogni idealizzazione della realtà è destinata ad allontanarsi dal reale in quanto tale. Sebbene tale passaggio sia ineludibile per la comprensione stessa del reale, occorre tener conto del referente ultimo dell'astrazione, senza dare a quest'ultima il primato. Ciò può avvenire nella vita del singolo, che sviluppa una teoria, spesso inconscia, sul mondo, come nelle concezioni teoretiche della filosofia e della teologia o dei fondamenti della concezione di società che guidano inconsapevolmente le scelte politiche. A tale attenzione ha richiamato recentemente papa Francesco nell'enciclica Evangelii Gaudium, dedicandovi un paragrafo intitolato "La realtà è superiore all'idea" (cf. EG 231). Rimandiamo a questo proposito alle interessanti riflessioni di G. PICCoLo, "La realtà è superiore all'idea. Il pensiero contemporaneo torna ad essere realista?", in Civiltà Cattolica 4011-4012/3 (2017) 298-304. 
sia Dio stesso".

Minus: "Per te amore e Dio sono la stessa cosa allora?".

David: "Questo pensiero è il solo conforto alla mia miseria ed alla mia disperazione".

Minus: "Continua papà".

David: "Di colpo la miseria è diventata ricchezza e la disperazione speranza. È come essere graziati Minus, in punto di morte".

Minus: "Papà, se è vero ciò che dici, allora Karin è tutta circondata da Dio perché noi l'amiamo davvero".

David: "Sî".

Minus: "Questo può aiutarla?".

David: "Penso di sì".

Il padre allora si allontana per preparare la cena. Minus rimane da solo e ancora incredulo per il dialogo avuto col padre, esclama: "Papà ha parlato con me!”. Su queste parole la scena viene oscurata e il film ha termine.

Analizzando il film dalla prospettiva soteriologica, riteniamo di poter individuare l'emergere di un bisogno di salvezza. Innanzitutto un bisogno di essere salvati dalla malattia. La drammatica schizofrenia di Karin pone con forza la domanda sul senso della sofferenza sia per lei stessa che per i suoi familiari. Questo bisogno di salvezza si declina anche come bisogno di dare senso a un esistenza che sembra essere irreale. Più volte, infatti, come già evidenziato altrove, si parla della difficoltà di vivere in un mondo altro da questo e, a motivo di ciò, di entrare in relazione con il mondo dell'altro. Sono i dubbi che Minus confida alla sorella: "Mi domando se tutti vivano chiusi in se stessi [...] nel proprio mondo. Tu nel tuo. Io nel mio". La separazione tra il reale e l'ideale è portata all'estremo nella schizofrenia di Karin: "La malattia era come un sogno. Ma questo non può essere un sogno. Deve essere la realtà! Deve essere la realtà! [...] A volte mi trovo in questo mondo a volte nell'altro, senza che io possa impedirlo". Ma anche David ha vissuto questo dramma scontrandosi con la realtà: "Vedi Karin si traccia un magico cerchio attorno a noi escludendo ciò che può compromettere i nostri intenti. Ma, quando la vita spezza il cerchio, questi intenti si rivelano meschini e insignificanti. Così tracciamo un nuovo cerchio e un nuovo riparo".

In tutti i casi, sembra potersi concludere che la salvezza non può trovarsi nell'astrattezza dalla realtà, lottando a tutti i costi per ben ancorarsi al cerchio confezionato da noi stessi. Fare i conti con la realtà è la strada per la 
salvezza, non solo del soggetto, che viene messo a nudo dall'esperienza, ma anche degli altri, che altrimenti risulterebbero irraggiungibili e intangibili, chiusi in altrettanti cerchi incomunicabili. Questo bisogno di comunicabilità emerge attraverso il giovane Minus, che vorrebbe fin dall'inizio interagire col mondo del padre. Diversi sono i tentativi infatti di intavolare un dialogo su questioni più profonde e vitali, ma David sembra sottrarvisi. Soltanto alla fine del film, nell'ultimo dialogo, l'interazione si realizza e padre e figlio comunicano. Dunque, anche qui, ritroviamo una salvezza che nasce dalla relazionalità, stavolta come comunicabilità intersoggettiva su una realtà di cui nessuno ha possesso, ma con cui ciascuno si trova a fare i conti in modo drammatico.

Il ruolo centrale del padre di Karin è nuovamente un richiamo ai temi dell'angoscia esistenziale e alla possibilità di una vita autentica. È di nuovo il suicidio la tentazione che incombe sul soggetto come rimedio ad una vita che non offre nulla ma solo nausea e angoscia. Proprio nel momento in cui la morte diviene sempre più vicina, ci racconta lo stesso protagonista, qualcosa irrompe nella sua vita per riorientarla. Il riconoscimento del suo egoismo di fronte alla morte della moglie o alla malattia di Karin, si trasforma nella consapevolezza della presenza di qualcosa che lo trascende. Sono le relazioni in cui l'amore si rivela: "Dal mio animo vuoto sbocciò qualcosa che non ho quasi il coraggio di nominare. Un amore per Karin, per Minus e per te".

Dunque per David, la nostalgia di salvezza si è declinata innanzitutto come salvezza dalla propria chiusura egoistica, di oltrepassamento del suo cerchio. Una salvezza che produce relazioni nuove e autentiche nella sua vita. Una salvezza che nasce come amore e che genera amore, ma non solo. Qui la salvezza sembra assumere una dimensione ancora ulteriore. La salvezza verrebbe a coincidere con l'amore, che nel dialogo finale col figlio Minus è identificato con Dio stesso: "Questo pensiero è il solo conforto alla mia miseria ed alla mia disperazione". Dio è la sua unica speranza, diremmo, biblicamente, che è la sua roccia, il suo punto stabile, nella continua destabilizzazione della vita. Per David è dunque Dio la sua salvezza, non tanto dopo questa vita, ma di questa stessa vita: "Di colpo la miseria è diventata ricchezza e la disperazione speranza".

\section{Nostalgia di una Salvezza Che VIENe da Dio}

Infine il terzo e ultimo film che passiamo in rassegna è Luci d'inverno 
girato nel 1963. Il titolo originario è completamente diverso: I comunicandi (Nattvardsgästerna). È importante sottolineare questo stravolgimento perché nel titolo italiano è sottesa un'interpretazione che è tutt'altro che immediata e di facile contestazione. La tematica è qui completamente esistenziale-religiosa. Ma ci è lecito parlare di luci nell'inverno vissuto dal protagonista?

Il protagonista è un pastore protestante, Tomas Ericsson. Il film si apre con la celebrazione della messa. A questa partecipano in particolare gli altri personaggi centrali del film: la maestra di scuola elementare Märta Lundberg, i coniugi Persson, Karin e Jonas, il sacrestano Algot e l'organista Fredrik. Nella celebrazione si rinviene l'assenza di passione e il freddo distacco del pastore dal rituale religioso, che rispecchia il gelido inverno in cui la trama è ambientata. I pensieri del pastore sono altrove. È esistenzialmente sconvolto. Da quando la moglie è deceduta si è ritrovato a dover fare i conti con una prospettiva fino ad allora inesplorata. La sua fede non corrisponde più al nuovo mondo che gli si è palesato. Dio è ormai lontano e non ha risposte per questa solitudine e angoscia. Ogni sua frase su Dio gli appare irreale, senza alcun riscontro con la vita quotidiana. La sofferenza esistenziale è amplificata dal malore fisico e dalle ripetute immagini del Cristo sofferente sulla croce.

Al termine della celebrazione il pastore incontra dapprima i coniugi Persson. La signora Karin, che è in attesa di un figlio, gli fa presente la depressione del marito che è tormentato dalla possibilità che $\mathrm{i}$ cinesi possano da un momento all'altro sganciare una bomba atomica e distruggere il mondo. Per questo chiede al pastore di fissare un appuntamento per parlargli a tu per tu e dissuaderlo dalle sue manie di persecuzione e di suicidio. Così a Jonas viene chiesto di ritornare in chiesa dopo una ventina di minuti, il tempo di accompagnare la moglie a casa.

Usciti dalla sacrestia giunge Märta, innamorata di Tomas, ma da questi rifiutata nonostante le continue dichiarazioni dell'amore che prova per lui. Per questo vive in uno stato di estrema sofferenza e tristezza, ma sa il motivo: "Non puoi sposarmi perché non mi ami". Märta si definisce atea e cerca di rimuovere in Tomas quelle resistenze che lo lasciano ancora tenuamente ancorato a una fede soprannaturale: "Il silenzio di Dio. Dio non dice niente. Dio non parla perché non esiste. Ė semplice!”. I due così si separano. Allora Tomas decide di leggere una lettera che Märta gli aveva scritto e fatto recapitare il 
giorno prima. Ma prima di farlo riguarda le foto della sua amata moglie.

La lettera è lunga e, per gli spettatori, viene letta in prima persona da Märta. Il testo è intenso e i sentimenti sono messi a nudo attraverso una profonda analisi introspettiva:

Io non ho mai creduto alla tua fede: la ragione principale deriva dal fatto che non sono mai stata tormentata da tentazioni religiose. Sono stata allevata in una famiglia agnostica. Dio e Cristo non esistevano che come vaghi significati. Quando venni in contatto con la tua fede, questa mi parve oscura e nevrotica, e in un certo qual modo pervasa di sentimenti crudeli e primitivi. [...] la vita è già complicata com'è senza fattori soprannaturali.

Gli racconta allora di quando, soffrendo per un eczema acuto a tutte e due le mani, si erano scontrati sulla possibilità di una preghiera che fosse capace di porre rimedio a un tale strazio da non farla più dormire durante la notte:

Ricordo che ti dissi: sta' zitto, ora prego io, dal momento che tu non puoi farlo. Dio, dissi fra me, perché mi hai procurata un'eterna scontentezza, paura e tanta amarezza? Perché devo capire la mia miseria? Perché devo soffrire come all'inferno per la mia indifferenza? Se hai uno scopo nella mia sofferenza, dimmelo, così potrò sopportare senza lagnarmi. Io sono forte, tu mi hai creata terribilmente forte e nello spirito e nel fisico, ma tu non assegni alcun compito alla mia forza. Dammi una ragione di vita e io diverrò la tua serva obbediente. Così, all'incirca, fu la mia preghiera.

La preghiera venne esaudita, sostiene la stessa Märta: "Avevo chiesto una luce e l'avevo avuta. Ho chiesto uno scopo e l'ho avuto. Quello scopo sei tu". Il dramma esistenziale è vistoso e senza possibilità di essere risolto. L'esistenza di Märta non ha alcun senso senza Tomas. Ma il pastore sa bene di non amarla e non può che rispondere in modo freddo, cercando di allontanarla.

Terminata la lettura giunge in sacrestia Jonas. La difficoltà esistenziale di Tomas è tale da sottoporre Jonas ad una personale e sincera confessione dello stato in cui si trova:

Da quando mia moglie è morta la vita mi sembrò finita. [...] Non avevo più motivi per continuare a vivere [...] Ma devo andare avanti non per me, per gli altri [...] Per caso divenni pastore [...] Io e il mio Dio 
vivevamo in un mondo fatto appositamente solo per noi [...] Non posso aiutare nessuno perché sono un cattivo pastore [...] Tutte le volte che ho messo Dio a confronto con la realtà l'ho visto diventare feroce, distante e crudele [...] Solo mia moglie sapeva vedere il mio Dio [...] Se veramente Dio non esistesse, nulla avrebbe più importanza. La vita avrebbe una spiegazione, sarebbe un sollievo, la morte è solo una frattura: la fine del corpo e dell'anima. La crudeltà della gente, la sua solitudine, i suoi timori, tutto sarebbe chiaro come la luce del giorno. Le sofferenze non dovrebbero più essere spiegate. Non esisterebbe un creatore né un tutore. Niente pensieri.

Jonas che si aspettava risposte alle sue preoccupazioni si ritrova ad ascoltare, a sua volta, una drammatica confessione e alla fine decide di andar via. Il pastore Tomas rimasto solo si rivolge ancor una volta al Dio di cui avverte un silenzio impenetrabile: "Dio perché mi hai abbandonato?". Nel frattempo in chiesa era tornata Märta. Mentre le si avvicina cade a terra, probabilmente per la forte febbre e, come liberato da un peso, le dice: "Sono libero, libero finalmente. Ho avuto una lieve speranza che non erano tutte ipotesi, sogni, bugie". Ma questa improvvisa luce durerà ben poco. Infatti subito dopo sopraggiunge una donna che avverte Tomas che il signor Persson si è suicidato con un colpo di fucile alla testa nei pressi di un torrente. Allora Tomas, che deve recarsi a celebrare la messa in una vicina chiesa, parte in macchina assieme a Märta. Si ferma poi sul luogo dell'accaduto per rivolgere un ultimo sguardo a Jonas. La domanda che riecheggia dal fragore delle acque del torrente nelle orecchie degli ascoltatori richiama alla possibilità che tale morte sia stata determinata dallo stesso vuoto esistenziale del protagonista, incapace di comunicare speranza attorno a sé.

Tomas riparte in auto con Märta, lungo il tragitto che lo condurrà alla celebrazione della messa che conclude il film. La spasimante lo invita a passare a casa sua per ricevere alcune medicine per alleviare il suo acuto stato influenzale. Suo malgrado, Tomas si reca a casa di Märta e l'attende nell'aula dove la maestra tiene le lezioni per i ragazzi. Mentre attende il ritorno, sopraggiunge un ragazzo che aveva dimenticato un quaderno in aula e viene incalzato da alcune domande del pastore che sembrano una sorta di interrogatorio puntuale, che conducono alle domande conclusive, le uniche che non avranno una risposta precisa, ma solo un vago "non lo so". Tomas aveva infatti chiesto al ragazzo se volesse entrare in seminario come aveva fatto il fratello più grande $\mathrm{e}$ se lo stesso fratello si trovasse bene in seminario. Bergman, in tal modo, lascia 
aperta la domanda sul parallelismo tra la desolazione raggiunta dal pastore e se, a ciò, non sia destinato anche il giovane seminarista.

Al ritorno di Märta inizia un altro dialogo, dove l'amore non corrisposto conduce la giovane maestra ad implorare di sposarla, Tomas oppone con distacco severi giudizi su di lei e sulla possibilità di amarla:

Non ti amo [...] Sono stanco delle tue premure, dei tuoi lamenti, dei tuoi consigli, delle tue attenzioni e dei tuoi regali. Sono anche stanco della tua miopia, della tua inettitudine, della tua timidezza, di tutte le tue stucchevoli espansioni. Mi obblighi senza darmi respiro a subire le tue inutili cure, la tua cucina, i tuoi malanni, le tue stanchezze, i tuoi sciocchi geloni. Devo andare lontano da questo pesante carico di affanni terreni.

L'accusa è pesantissima. Tomas le contrappone la figura della sua defunta moglie, di cui non potrà mai prendere il posto: "Mia moglie l'ho amata. Non amo te perché ho amato mia moglie. Con lei sono morto anche io. [...] L'ho amata ed era tutto ciò che tu non riuscirai mai ad essere". Giunge addirittura a dirle che è inutile cercare di imitarla. Ma timidamente Märta gli obietta di non averla mai conosciuta. Il volto di Tomas allora si riempie di perplessità e si impone di non continuare per non esagerare ulteriormente. Märta, piangente e singhiozzante, controbatte: "Tu da solo affonderai e nulla riuscirà a salvarti. Finirai per odiare la vita anche più di te stesso".

Nonostante ciò, Tomas invita Märta a ripartire con lui. Prima di giungere alla parrocchia Tomas si reca a casa della vedova, per comunicarle la triste notizia del suicidio del marito. Karin si ritrae in disparte, mentre i suoi tre figli sono a tavola, e, all'ascolto della morte del marito, si accascia sui gradini delle scale di casa, ponendo una mano sul pancione, come a proteggere il figlio che deve ancora nascere e tragicamente riconosce: "Allora sono sola". Tomas le propone di recitare assieme una preghiera, ma si rifiuta, poiché il suo pensiero va ai bambini a cui dovrà comunicare la drammatica morte del padre. Karin si mostra come rassegnata a un destino che sapeva imminente e infine ringrazia il pastore dicendogli che sicuramente ha fatto tutto quello che ha potuto per aiutare il marito. Tomas avverte ancor una volta di essere inadeguato e nauseato da una vita che non comprende.

Giunto in chiesa incontra lo storpio sacrestano Algot che lo attendeva per parlargli. Gli si siede accanto e dice di volerlo fare partecipe di una sua teoria sulla passione di Cristo: 
La sofferenza di Cristo si interpreta male [...] Si pensa troppo alla tortura, per così dire, che non deve essere poi stata talmente atroce. Sì, mi scusi; naturalmente sembra presuntuoso, ma fisicamente, modestia a parte, io ho sofferto tanto quanto Cristo. Le sue sofferenze furono di breve durata [...] Io credo che ci sia stata una sofferenza ben più grande a quella fisica [...] Forse mi sbaglio. Ma pensi al Getsemani, pastore. Tutti i discepoli si addormentarono, non avevano capito niente, neanche l'ultima cena, niente di niente. E quando i soldati arrivarono, quelli se la diedero a gambe [...] Per tre anni Gesù aveva parlato con questi discepoli, erano vissuti insieme ogni giorno. Ma quelli semplicemente non avevano capito ciò che aveva detto [...] Tutti lo abbandonarono e lui rimase solo. Pastore, questa deve essere stata una vera sofferenza! Sapere che nessuno aveva capito. Essere abbandonato quando più si ha bisogno di qualcuno in cui aver fiducia. Una sofferenza terribile [...] Ma doveva esserci di peggio! Quando Cristo fu inchiodato alla croce e se ne stava là appeso con la sua sofferenza, gridò: "Dio, Dio mio, perché mi hai abbandonato?”. Gridò con quanto fiato aveva in gola. Come se avesse creduto che suo Padre nei cieli l'avesse abbandonato. Lui credette che tutto quello che aveva predicato fosse menzogna! Cristo fu preso da un grande dubbio negli istanti prima di morire. E questa deve essere stata la sua più orribile sofferenza. Intendo dire: il silenzio di Dio. Non è vero, pastore?.

Tomas non può che rispondere un flebile: "Sì, sì". È una confessione rimandata fin dall'inizio del film e che, proprio ora, prima della celebrazione della messa posta a conclusione, sembra riflettere e attualizzarsi nel vissuto esistenziale di Tomas. La sua debole salute fisica è di fatto nulla rispetto alla sua profonda solitudine esistenziale. Si ritiene abbandonato, se non fisicamente, almeno in quella forma di vicinanza empatica, che nessuno forse sa più mostrargli dopo la morte della moglie. E infine la forma più eloquente, già menzionata dallo stesso protagonista, del vivere l'abbandono di Dio, il suo silenzio, come abbandono di tutte le sue certezze, di tutto ciò in cui aveva creduto e che è a fondamento del suo ruolo sociale, il quale viene inevitabilmente a disgregarsi e a cedere.

Ma ancora una scena, a nostro giudizio, profondamente espressiva sta per accadere in chiesa. L'organista, in uno stato di ubriachezza, si avvicina a Märta e le rivela la sua insoddisfazione per l'operato del pastore, che con il suo fare, a suo dire, ha causato l'allontanamento dei fedeli o, forse, del pubblico che poteva offrire un qualche riconoscimento al musicista:

Guarda che cosa ha fatto Tomas. La gente affollava la chiesa allora. Ah, 
era sua moglie che la tirava. Adesso si tappa le orecchie. Sì, sua moglie, sì. E Tomas non ha la minima idea della natura umana, vedeva solo lei, viveva per lei, era innamorato pazzo di lei. Cara Märta, quante storie per quell'amore. Dio è l'amore e l'amore è Dio, l'amore è una prova dell'esistenza di Dio, l'amore, la sola realtà di questo nostro pietoso mondo terreno. Le so a memoria. Sono stato a sentirle tutte quante le sue prediche. Dammi retta, bambina mia, scappa finché sei in tempo.

Al suono dell'ultima campana, che avvisa l'inizio della messa, Märta cade in ginocchio in un banco e, come se desiderasse vedere qualcosa che non vede, sussurra: "Se riuscissimo ad essere sicuri [...] Se riuscissimo a credere in una verità. Se riuscissimo a credere". Ha così inizio la celebrazione finale, in una chiesa ancora più vuota di quella in apertura. Stavolta vi sono soltanto Märta, Algot e l'organista. Il film termina con l'incipit della messa e Tomas che invoca: "Santo, Santo, Santo, il Signore Dio degli eserciti. Benedetto colui che viene nel nome del Signore".

L'intero film è attraversato da ciò che il pastore Tomas identifica col silenzio di Dio e col riconoscimento di una fede che non corrisponde alle esigenze reali ed esistenziali del suo io più profondo. C'è sicuramente in lui un bisogno di qualcosa che lo liberi dalle angosce esistenziali, dai tormenti di una vita senza senso. Anche qui potremmo parlare, come per il professor Borg, del bisogno di una vita autentica. È una vita nascosta agli occhi del pastore fino alla scomparsa della moglie. In quel momento percepisce un abisso tra la sua realtà di pastore e la possibilità di una fede che dia risposte sensate ai drammi umani.

L'unico pensiero che sembra tenerlo in vita è di andare avanti non per sé ma per gli altri, come sostiene nel colloquio con Jonas. L'infinita lontananza e l'assenza che avverte del suo Dio hanno ridotto il suo ministero pastorale all'unica dimensione per lui visibile: quella di un impegno sociale. Ma anche questo barcolla incredibilmente quando viene a conoscenza che Jonas si è suicidato subito dopo l'incontro avuto con lui. Anche la dimensione dell'essere per gli altri sembra cedere e la diminuzione delle persone presenti all'ultima celebrazione ne è il segno.

Possiamo dunque parlare di luci? C’è nel pastore una qualche apertura a una concreta salvezza? E non ci riferiamo esclusivamente a una salvezza 
dopo la morte, ma alla possibilità di una salvezza in questa stessa vita. Guardando il volto di Tomas nel finale del film ci sembra di dover rispondere negativamente a entrambi gli interrogativi. Ancora in lui la vita procede senza senso. Tuttavia rimane nello spettatore il dubbio: anche il pastore giungerà al suicidio come Jonas? Oppure troverà nelle parole del semplice sacrestano una risposta al silenzio di Dio e una luce nel suo inverno?

Il pastore rimane dunque nel suo dramma esistenziale e nella nostalgia della salvezza, che potremmo nel suo caso identificare prima di tutto come nostalgia di verità, che sembra apparire soffusamente e per brevissimo tempo: "Sono libero, libero finalmente. Ho avuto una lieve speranza che non erano tutte ipotesi, sogni, bugie". Ė quella stessa nostalgia presente anche in Märta: "Se riuscissimo a essere sicuri [...] Se riuscissimo a credere in una verità. Se riuscissimo a credere". Una tale richiesta di fede, ma prima ancora di senso, non può trovare risposta in meri momenti sensazionalistici, destinati a essere lampi nel buio che presto svaniscono, ma in esperienze reali concrete, che a questo senso possono avvicinarsi se la sofferenza non conduce all'implosione del soggetto, ma a un'apertura alla condivisione, al riconoscimento dell'altro come interlocutore attivo e pertanto esperienzialmente già partecipe alla stessa sofferenza.

In linea con tale prospettiva, una risposta di salvezza ci sembra ravvisabile nella figura del sacrestano Algot. Per Tomas all'inizio del film appare come un uomo distante dai suoi dubbi esistenziali. La sua tranquillità del vivere sembra essere motivata dall'ignorare quei problemi che incombono sul pastore. Ma non è per nulla così, anzi a partire dalla sua sofferenza fisica, comprende che può esserci un male ben più grande. La sua riflessione sulla passione di Cristo giunge a una conclusione che lascia come impietrito il pastore per la profondità dell'analisi: la sofferenza più grande non è né quella fisica né l'abbandono degli altri, ma il silenzio di Dio stesso. È qui che ci sembra di poter intravedere la possibilità di una salvezza come comunione e comprensione dell'io più profondo. Nessuno sembra essere in grado di entrare nelle angosce esistenziali del pastore o, almeno, questo lui ritiene: è da solo di fronte al suo io, dopo che la sua amata moglie è morta. Non vi riesce Märta che soffre per l'amore non contraccambiato. Non poteva comprenderlo Jonas con cui, forse proprio per questo, Tomas si era completamente messo a nudo nel dialogo in sacrestia prima del suicidio. Non lo capiva Karin che lo riteneva capace di aiutare il marito a distoglierlo dalle sue manie. Tuttavia, lo sguardo rasserenato e penetrante al 
tempo stesso del sacrestano Algot sembra lasciar trasparire questa consapevolezza profonda: ha compreso il dramma di Tomas e, in modo sottile, cerca di comunicarglielo. Il film ci sembra accentuare proprio questo sentimento di assenza di comunione tra gli esseri umani, incapaci di comprendere le difficoltà esistenziali più profonde dell'altro, lasciando tuttavia la questione aperta.

Sebbene questo stato di solitudine non sia attribuibile all'abbandono diretto degli uomini come nel caso di Gesù di Nazareth, analizzato da Algot, non se ne sminuisce l'effetto, anzi si acuisce ulteriormente annullando la colpevolezza altrui. Rimane tuttavia l'apice dell'incomprensione e della solitudine, che nel caso del pastore, è strettamente connessa al silenzio di Dio. Ma, allora, se l'angoscia più profonda si manifesta in questa assenza, riemerge in contrasto la possibilità di una salvezza che nasce proprio dal parlare di Dio e da una sua autocomunicazione più profonda di ciò a cui l'uomo anela e di cui avverte un senso di estrema nostalgia. Tale aspetto richiama in qualche modo la riflessione heideggeriana secondo cui "solo un Dio ci può salvare"11. $\mathrm{Da}$ un lato per il bisogno di apertura verso un oltre da attendere per innovare e ridonare vita ad una realtà angosciante, ma, dall'altro lato, diremmo noi, proprio in quanto le profondità abissali della solitudine e dell'assenza di Dio non sono ignote a Dio stesso o, per dirla agostinianamente, è solo Lui che conosce la parte più intima e impenetrabile del cuore umano con tutte le sue inquietudini.

La profondità dell'analisi che Bergman offre della angosciosa solitudine del protagonista di questo film, ma anche degli altri personaggi di questo e di altri suoi film, espone con particolare intuito quanto messo in luce dalla filosofia esistenzialista in opposizione alle correnti neopositiviste, che avrebbero cercato di ridurre l'uomo stesso ad una serie di impulsi e di passioni da passare al vaglio esclusivo della psicanalisi. La domanda che emerge è dunque se tale angoscia esistenziale sia riconducibile e riso-

11 M. Heidegger, Ormai solo un Dio ci può salvare. Intervista con lo "Spiegel" (Ugo Guanda Editore, Parma 1987) 284. Riportiamo di seguito uno stralcio dell'intervista ad Heidegger del 23 settembre 1966 da parte della rivista settimanale Der Spiegel in cui il filosofo tedesco espone il suo pensiero su una salvezza che viene da Dio: "La filosofia non potrà produrre nessuna modificazione immediata dello stato attuale del mondo. E questo non vale solo per la filosofia, ma per ogni riflessione e per ogni aspirazione degli uomini. Solo un Dio, ormai, può aiutarci a trovare una via di scampo. Vedo, come unica possibilità di via di scampo, questo: preparare, nel pensiero e nella poesia, una disponibilità e una prontezza per l'apparizione del Dio oppure per l'assenza, il dis-stanziarsi, del Dio nel tramonto; in modo che il nostro destino non sia quello, per dirla brutalmente, di "crepare», ma che sia, se dobbiamo tramontare, quello di tramontare al cospetto del Dio assente". 
lubile nell'alveo delle scienze psicologiche o vi sia dell'altro e dell'oltre. Ad approdare a questo secondo versante sono le considerazioni che pone Romano Guardini a proposito della malinconia di fondo di quanti si mostrano particolarmente sensibili alle sofferenze, come apertura e richiamo alla nostalgia di $\mathrm{Dio}^{12}$ :

Medici e psicologi ti sanno dire un mucchio di cose, e tutte pertinenti, circa le cause e la struttura intima della malinconia. Purtuttavia e spesso vi frappongono cose talmente banali, che non si sa proprio come mandarle d'accordo con la profondità e la violenza della passione che sta sotto a quella esperienza. Ciò che essi ti sanno dire non va oltre la teoria di certe sottostrutture fondamentali. Il vero significato non si rivela se non attraverso lo spirito. E mi pare che lo si debba formulare così: la malinconia è l'inquietudine dell'uomo che avverte la vicinanza dell'infinito ${ }^{13}$.

A noi sembra che proprio a tale comprensione sia giunto lo storpio sacrestano, penetrando le inquietudini di Tomas, ma al tempo stesso rasserenato da qualcosa d'altro, come avvolto da una saggezza nascosta che sa guardare oltre l'apparenza del silenzio di Dio, riapprodando a un senso profondo dell'esistere umano, che in qualche modo è già parola di Dio.

\section{ALCUNE RIFLESSIONI CONCLUSIVE}

Lesistenzialismo cinematografico di Bergman nei tre film passati in rassegna mostra un intenso impegno di riflessione filosofica e di introspezione psicologica, ricercata nella cura ai dettagli, visivi e sonori, come nella ricerca di trasmissione del vissuto interiore dei protagonisti. In essi le attese di salvezza si svelano come ricerca di autenticità e di senso e le loro angosce esistenziali mostrano, a nostro giudizio, una nostalgia di salvezza nei confronti di una vita che genera nausea e un forte senso di disagio. È il bisogno di

12 Di questa nostalgia ne ha parlato papa Francesco durante l'omelia del 6 gennaio 2017: "I magi [...] esprimono il ritratto dell'uomo credente, dell'uomo che ha nostalgia di Dio; di chi sente la mancanza della propria casa, la patria celeste. Riflettono l'immagine di tutti gli uomini che nella loro vita non si sono lasciati anestetizzare il cuore. La santa nostalgia di Dio scaturisce nel cuore credente perché sa che il Vangelo non è un avvenimento del passato ma del presente. [...] La nostalgia di Dio ha le sue radici nel passato ma non si ferma lì: va in cerca del futuro. Il credente "nostalgioso", spinto dalla sua fede, va in cerca di Dio, come i magi, nei luoghi più reconditi della storia, perché sa in cuor suo che là lo aspetta il Signore" (cf. http://w2.vatican. va/content/francesco/it/homilies/2017/documents/papa-francesco_20170106_omelia-epifania.html).

13 R. Guardini, Ritratto della malinconia (Morcelliana, Brescia 1952) 54-55. 
qualcosa che dia speranza per resistere alla continua tentazione del suicidio, affrettando quella morte che sarebbe l'unica possibilità di attuazione certa, con cui o si fanno i conti in questa vita, accettando il rischio dell'angoscia, oppure si continua a vivere nell'inautenticità.

Queste ricerche introspettive ed esistenziali non sempre conducono a un approdo, ma neppure dissolvono il problema come inutile o insignificante. Il superamento dell'esistenzialismo, quando è compiuto nell'annullare i dubbi e gli interrogativi, si separa dalla realtà, dalla profondità del proprio essere, e il rinchiudersi nelle tacite ideologie può degenerare nella superficialità della ricerca dell'avere e dell'apparire. I personaggi di Bergman: il professore Borg, il pastore Tomas e il padre di Karin, David, si sono trovati a scontrarsi con una vita vissuta a livello dell'inautentico, ma si sono fatti carico del peso della ricerca esistenziale. Il bisogno di rispondere autenticamente si è manifestato come insopprimibile. Per questo il bisogno di salvezza non è stato evaso. Hanno accettato intimamente di non poter farne a meno, anche quando questa salvezza non è stata in alcun modo visibile.

La salvezza si è declinata innanzitutto come salvezza in questa vita nella ricerca di relazioni interpersonali, nella prospettiva del vivere per gli altri, nella possibilità di amare gratuitamente. Non sono approdi immediati né tanto meno capaci di dissolvere gli interrogativi esistenziali. Sono più comprensibili come lotta e resistenza di fronte all'angoscia esistenziale che sembra continuare ad incombere e potrebbe ancora avere la meglio. Infine, quando la salvezza viene intesa come stabilità in una vita in balia delle onde del reale, essa viene riconosciuta nella presenza di Dio come garante dell'autenticità di ogni atto e desiderio di amore. Anche tale intuizione di una salvezza che viene da Dio potrebbe essere interpretata come un'immagine confusa, dai contorni distorti, come nello specchio paolino. Tuttavia la distorsione dello specchio presuppone la riflessione di una realtà, quella della sofferenza che non si rassegna al non senso, ma invita a cercare sempre oltre, perché di quest'oltre avverte una profonda nostalgia ${ }^{14}$, al di là di ogni idealizzazione deformante che non può mai del tutto annullare ciò che si cela nella realtà stessa: la parola silente e salvifica di Dio.

14 Sulla dialettica della ricerca e del cercare sempre oltre, in relazione alla fede come amore che spinge ad incamminarsi verso un amore sempre più intenso, rimandiamo all'introduzione di G. Mazzillo, L'uomo sulle tracce di Dio. Corso di Introduzione allo studio delle religioni (Edizioni Scientifiche Italiane, Napoli 2005) e ad un approfondimento più recente G. MazzILlo, "La fede tra dono e ricerca", in Vivarium 20/3 (2012) 241-262. 
Al termine di queste riflessioni, ci piace riportare una citazione di Karl Rahner, in cui il movimento che abbiamo cercato di descrivere in modo più analitico, assume i tratti della poesia e della preghiera e sembra porsi proprio in dialogo coi protagonisti dei film di Bergman su cui ci siamo soffermati in questo lavoro:

Potrebbe il mio piccolo nido, l'angusto mondo delle mie cose familiari, questa vita terrena, con le sue grandi gioie e i grandi dolori, potrebbe essermi patria se non riposasse tutto nell'abbraccio della tua lontana infinità? Mi sarebbe mai patria la terra se non le si stendesse sopra il cielo tuo lontano? E volessi pure ostinarmi a credere solo nel mio essere finito, a riconoscervi l'unico senso della mia vita, come tanti fanno, e lo professano apertamente, dove troverei la chiarità dello spirito che accetta consapevole questa finitudine come unica sua sorte, se non avessi levato prima lo sguardo a quell'orizzonte lontano dove è l'inizio dell'essere tuo infinito? Ché questo mio piccolo essere affonderebbe nel buio e nell'inconscia sua piccolezza, senza dolore di nostalgia, senza coscienza di rassegnazione, se la luce dello spirito non si potesse spingere oltre i propri limiti, nello spazio senza confini, che tu, silenzioso infinito, riempi. Dove rivolgermi dunque, per sfuggire a te, se il coraggio della mia finitudine, come la brama nostalgica dell'infinito non fanno che confessare te? Che ti posso più dire di te se non so che tu sei quello senza cui io non posso essere, che tu sei l'infinito in cui solo può vivere la mia finita umanità? ${ }^{15}$.

Non possiamo che concludere queste riflessioni con una considerazione di Giovanni Mazzillo, a commento e sintesi proprio dei versi appena citati, come a partecipare a un dialogo e a una ricerca mai conclusa in se stessa, ma non per questo inconcludente e senza raggiungimenti o incontri con la Realtà che è continuamente cercata, che plasma e che attrae verso Sé, con la stessa forza, paradossalmente attrattiva e repulsiva al tempo stesso, con cui spinge verso gli altri, in un'apparente lontananza, attraverso cui in modo inatteso si realizza invece l'incontro: "Potrei respirare l'ebbrezza di infinito nella mia limitatezza e finitudine, se Tu, mio Dio, non fossi qui, se non fossi anche adesso con me. Tu, il silenzioso e nostalgico Infinito che preme dentro di me?" ${ }^{16}$

15 Cf. K. Rahner, Visioni e profezie. Mistica ed esperienza della trascendenza (Vita e pensiero, Milano 1995).

16 La citazione è presente in una nota a margine di alcune dispense per il Corso "Semina Verbi" nella post-modernità? Nuove opportunità di evangelizzazione" tenuto da Giovanni Mazzillo neIl'anno accademico 2015/2016 presso l'Istituto Teologico Calabro per la licenza in Teologia dell'Evangelizzazione(http://www.puntopace.net/DISPENSE/LICENZA/Postmoderno-Evangelizzazione/VisioneScientifica DelMondodo.pdf). 